\title{
Summary of the National Advisory Committee on Immunization's Update on quadrivalent meningococcal vaccines available in Canada
}

\author{
Henry $B^{1,2}$, on behalf of the National Advisory Committee on Immunization* \\ ${ }^{1} \mathrm{NACl}$ Meningococcal Working Group Chair \\ ${ }^{2}$ Deputy Provincial Health Officer, Ministry of Health, Victoria, BC \\ *Correspondence: naci-ccni@phac-aspc.gc.ca
}

\begin{abstract}
Background: Invasive meningococcal disease (IMD) has an overall mortality of approximately $10 \%$ and up to $35 \%$ of survivors may experience long term sequelae. Canada's National Advisory Committee on Immunization $(\mathrm{NACl})$ recommends immunization with a quadrivalent conjugate meningococcal vaccine of individuals who are at increased risk of IMD due to an underlying medical condition or have a high risk of exposure to $N$. meningitidis. Use of a conjugate vaccine, either monovalent or quadrivalent, is recommended for a routine adolescent booster dose at around 12 years of age.

Objective: To review and update the evidence on the use of quadrivalent (serogroups A, C, Y and W-135) conjugate meningococcal vaccines and vaccination schedules used in Canada following the approval of a new quadrivalent meningococcal vaccine conjugated to the tetanus toxoid (Men-C-ACYW-TT, Nimenrix ${ }^{\mathrm{TM}}$ ).

Methods: NACl reviewed the knowledge synthesis performed by the Meningococcal Working Group, including information on the IMD burden of disease; safety, immunogenicity, efficacy and effectiveness of the new vaccine; currently used vaccine schedules; and other aspects of the overall immunization strategy. Following the review of evidence, $\mathrm{NACl}$ voted on specific recommendations.
\end{abstract}

Results: A total of 21 studies were reviewed for immunogenicity and 12 studies for safety data relating to Men-CACYW-TT. Information from two additional studies assessing immunogenicity and safety of a quadrivalent meningococcal vaccine conjugated to the diphtheria toxoid (Men-C-ACYW-DT, Menactra ${ }^{\circledR}$ ) in children 9 and 12 months of age was also reviewed. A good immune response and an acceptable safety profile when compared to the monovalent conjugate meningococcal vaccines (Men-C-C) were demonstrated by both vaccines in all the reviewed studies. For children less than 2 years of age, $\mathrm{NACl}$ continues to recommend the use of Men-C-ACYWCRM (Menveo ${ }^{\mathrm{TM}}$ ) vaccine.

Conclusion: The new and complete set of current recommendations for conjugate meningococcal vaccines will be published in the updated "Meningococcal" chapter in the Canadian Immunization Guide in the near future. The top surveillance and research priorities are: determining the coverage and impact of immunization (including carriage and herd immunity) on IMD in Canada; determining the duration of protection/immunity to allow the assessment of recommendations for booster doses of conjugate quadrivalent vaccines; comparative studies of the three available quadrivalent conjugate vaccines in the general population and high-risk groups; and the immunogenicity and safety of co-administration of quadrivalent vaccines with routine age appropriate vaccines, including the newly authorized meningococcal $B$ vaccine. 
Updated NACI recommendations on quadrivalent meningococcal vaccine (1)

\section{Recommendation \#1}

For routine immunization of adolescents, any of the quadrivalent or monovalent $C$ conjugate meningococcal vaccines registered in Canada may be used. The choice between quadrivalent and monovalent $\mathrm{C}$ conjugate vaccines is dependent on local epidemiology and other programmatic considerations. (NACl Recommendation Grade B)

\section{Recommendation \#2}

For the immunization of high-risk individuals 2 years of age and older, any of the quadrivalent conjugate meningococcal vaccines registered in Canada may be used. (NACl Recommendation Grade B)

\section{Recommendation \#3}

For the immunization of high-risk individuals between 8 weeks and less than 2 years of age, Men-C-ACYW-135 (Menveo ${ }^{\mathrm{TM}}$ ) is the recommended product. Schedules are provided in Table 3 of the "Meningococcal" chapter of the Canadian Immunization Guide (2). (NACl Recommendation Grade B)

\section{Recommendation \#4}

For immunization of individuals 2 years of age and older travelling to areas where meningococcal vaccine is recommended, any of the quadrivalent conjugate meningococcal vaccines may be used. (NACl Recommendation Grade B)

\section{Conflict of interest}

None

\section{References}

(1) National Advisory Committee on Immunization (NACl). Update on Quadrivalent Meningococcal Vaccines available in Canada: An Advisory Committee Statement (ACS). 2015 April. http://publications.gc.ca/site/eng/9.629655/publication.html

(2) National Advisory Committee on Immunization (NACI). Canadian Immunization Guide. 2015. http://www.phac-aspc.gc.ca/publicat/cig-gci/p04-meni-eng.php. 